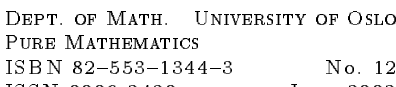

ISSN 0806-2439 $\quad$ June 2002

\title{
White Noise of Poisson Random Measures
}

\author{
Bernt $\emptyset \mathrm{ksendal}^{1,2}$ and Frank Proske ${ }^{1}$
}

June 7 th 2002

\begin{abstract}
We develop a white noise theory for Poisson random measures associated with a Lévy process. The starting point of this theory is a chaos expansion with kernels of polynomial type. We use this to construct the white noise of a Poisson random measure, which takes values in a certain distribution space. Then we show, how a Skorohod/Itô integral for point processes can be represented by a Bochner integral in terms of white noise of the random measure and a Wick product. Further, we apply these concepts to derive a generalized Clark-Haussmann-Ocone theorem for Lévy processes. Finally, an application of this theorem to portfolios in financial markets, driven by Lévy processes, is given.

Key words and phrases: Lévy processes, white noise, stochastic derivatives, chaos expansions, generalized Clark-Haussmann-Ocone formula, portfolios in financial markets

AMS (2000) Classification: primary 60H40; secondary 60G51,60G57,60H07, 91B28.
\end{abstract}

\section{Introduction}

An infinite dimensional analysis for Gaussian processes based on white noise was first treated by Hida $[\mathrm{H}]$ and then developed further by him and other researchers. See [HKPS] and the references therein. This approach provides a flexible and intuitive framework for a variety of applications. From the very beginning this theory has been used in quantum physics. See for example $[\mathrm{AH}-\mathrm{K}]$ or $[\mathrm{AHPS}]$, to mention a few. Later on white noise theory was applied to study stochastic partial differential equations [HØUZ] and more recently it has proved to be useful for applications in finance [AØPU]. Similarly a white noise theory has been developed for fractional Brownian motion and applied to finance [HØ1]. See also the generalization in $[\mathrm{EvdH}]$.

The object of this paper is to develop a white noise theory for Poisson random measures associated with Lévy processes. As an application we use this theory to prove a generalized version of the Clark-Haussmann-Ocone theorem (CHO) for Lévy processes [L] in terms of the white noise of a Poisson random measure. We apply then this formula to portfolios in financial markets driven by Lévy processes.

An extension of white noise theory to non-Gaussian analysis was already introduced in $[\mathrm{AKS}]$ and later generalized by [KDSU], [KDS]. The starting point of this theory is a biorthogonal decomposition, which extends the chaos expansion in Gaussian analysis. In a

\footnotetext{
${ }^{1}$ Department of Mathematics, University of Oslo, P.O. Box 1053 Blindern, N-0316 Oslo, Norway.

${ }^{2}$ Norwegian School of Economics and Business Administration, Helleveien 30, N-5045 Bergen, Norway.

E-mail addresses: oksendal@math.uio.no; proske@math.uio.no
} 
special case of their results the authors consider generalized Charlier polynomials and study Gel'fand triples, generated by these polynomials. They cover the compound Poisson noise, by using an unitary isomorphism between Poisson and compound Poisson spaces. In a different context and with another scope [DØP] develop a white noise theory for Lévy processes and use it to derive a generalization of the Clark-Haussmann-Ocone formula given in [LSUV]. Further, [Ka] introduces Skorohod integration by means of an Appell-like system of polynomials and apply it to certain classes of stochastic differential equations. In [BL] an anticipative calculus for Lévy processes is developed and applied to stochastic differential equations, too.

The paper is organized as follows: In section 2 we review some basic results on Lévy processes and the chaos expansion of Itô. We recall the construction of a Lévy process $\eta(t)$ from a given Lévy measure $\nu$, by means of the Bochner-Minlos theorem.

In section 3 we use a known chaos expansion for Lévy processes to define white noise $\stackrel{\tilde{N}}{(t, x)}$ of the compensated Poisson random measure $\tilde{N}(d t, d x)$, associated with $\eta(t)$. Then we define the Lévy Wick product $\diamond$ and introduce a Skorohod integral with respect to Poisson random measures for random fields $Y(t, x)$

$$
\int_{\mathbb{R}_{+}} \int_{\mathbb{R}} Y(t, x) \tilde{N}(\delta t, d x)
$$

and we prove that under some conditions we have

$$
\int_{\mathbb{R}_{+}} \int_{\mathbb{R}^{R}} Y(t, x) \tilde{N}(\delta t, d x)=\int_{\mathbb{R}_{+}} \int_{\mathbb{R}} Y(t, x) \diamond \stackrel{\dot{\widetilde{N}}}{ }(t, x) \nu(d x) d t .
$$

The Skorohod integral extends the Itô integral for point processes in the sense that both integrals will coincide if the the process $Y(t, x)$ is predictable, i.e. we have then

$$
\int_{\mathbb{R}_{+}} \int_{\mathbb{R}} Y(t, x) \tilde{N}(\delta t, d x)=\int_{\mathbb{R}_{+}} \int_{\mathbb{R}^{R}} Y(t, x) \tilde{N}(d t, d x) .
$$

In section 4 we recall a previously studied stochastic derivative operator $D_{t, x}$ and we prove a generalized $\mathrm{CHO}$ theorem.

\section{Preliminaries}

In this section we briefly recall some concepts and results in $[\mathrm{D} \emptyset \mathrm{P}],[\mathrm{L}]$.

Just as in the classical white noise theory it is convenient to choose a specific white noise as the underlying space, which enables an explicit construction of the Lévy process we are dealing with.

For general information about Lévy processes see $[\mathrm{B}],[\mathrm{P}]$ and $[\mathrm{Sa}]$. It is common to define a Lévy process $\eta(t)$ as a stochastic process on $\mathbb{R}_{+}$(or $\mathbb{R}$ ) with stationary independent increments and with $\eta(0)=0$. It can be shown that such a process has a cadlag version, which is then chosen to represent $\eta(t)$. In the following we are primarely interested in pure jump Lévy processes without drift. Such processes can be constructed explicitly as follows (for details see $[\mathrm{D} \emptyset \mathrm{P}])$ :

Let $\Omega=\mathcal{S}^{\prime}(\mathbb{R})$ be the Schwartz space of tempered distributions on $\mathbb{R}$. $\mathcal{S}^{\prime}(\mathbb{R})$ is the dual 
of the Schwartz space of test functions $\mathcal{S}(\mathbb{R})$ (see for definitions [GV]). We define the Lévy white noise probability measure $\mu$ on the Borelian sets $\mathcal{B}(\Omega)$ in virtue of the Bochner-Minlos theorem by the property

$$
\int_{\Omega} e^{i\langle\omega, f\rangle} d \mu(\omega)=e^{\int_{\mathbb{R}} \psi(f(y)) d y} ; f \in \mathcal{S}(\mathbb{R}),
$$

where $\psi$ is the characteristic exponent of a driftless pure jump Lévy process with Lévy measure $\nu$, given by

$$
\psi(u)=\int_{\mathbb{R}}\left(e^{i u x}-1-i u x \chi_{\{|x|<1\}}\right) \nu(d x)
$$

and where $\langle\omega, f\rangle=\omega(f)$ denotes the action of $\omega \in \mathcal{S}^{\prime}(\mathbb{R})$ applied to $f \in \mathcal{S}(\mathbb{R})$. We assume

that the Lévy measure $\nu$ satisfies

$$
M:=\int_{\mathbb{R}} x^{2} \nu(d x)<\infty
$$

From (2.1) and (2.3) we deduce that

$$
E_{\mu}[\langle\cdot, f\rangle]=0 \text { and } E_{\mu}\left[\langle\cdot, f\rangle^{2}\right]=M \int_{\mathbb{R}} f^{2}(y) d y ; f \in \mathcal{S}(\mathbb{R}),
$$

where $E_{\mu}$ denotes the expectation with respect to $\mu$. This isometry allows us to construct the driftless pure jump Lévy process $\eta(t, \omega)$ as the cadlag version of $\widetilde{\eta}(t, \omega)=\left\langle\omega, \chi_{[0, t]}(\cdot)\right\rangle$, which exists in $L^{2}(\mu)$, where

$$
\chi_{[0, t]}(s)=\left\{\begin{array}{l}
1 \text { if } 0 \leq s \leq t \\
-1 \text { if } t \leq s \leq 0, \text { except } s=t=0 . \\
0 \text { otherwise }
\end{array}\right.
$$

From now on we will work with this Lévy process.

We consider the filtered probability space

$$
(\Omega, \mathcal{F}, \mu),\left(\mathcal{F}_{t}\right)_{t \geq 0}
$$

where $\mathcal{F}_{t}=\mathcal{G}_{t} \vee \mathcal{N}$ is the filtration with $\mathcal{G}_{t}$ the $\sigma$-algebra generated by $\eta$ up to time $t$, and $\mathcal{N}$ the collection of all $\mu$-null sets, and where $\mathcal{F}=\mathcal{F}_{\infty}$.

The Lévy process $\eta$ can then be expressed by

$$
\eta_{t}=\int_{0}^{t} \int_{\mathbb{R}} x \tilde{N}(d s, d x)
$$

where $\tilde{N}(d s, d x)=N(d s, d x)-\nu(d x) d s$ denotes the compensated Poisson random measure associated with $\eta$. Here $N(d s, d x)$ is the Poisson random measure defined by

$$
N(\Delta t, \Lambda)=\sum_{s \in \Delta t} \chi_{\Lambda}\left(\Delta \eta_{s}\right) .
$$


See e.g. $[\mathrm{P}]$ for more details.

Next we recall a chaos expansion for square integrable functionals of $\eta$. This result can be found in $[\mathrm{I}]$. See $[\mathrm{L}]$ for a different proof. The chaos expansion is in terms of iterated integrals with respect to the compensated Poisson measure $\widetilde{N}(d s, d x)$.

In the following we still keep the integrability condition (2.3) and we denote by $\lambda$ the Lebesgue measure on $\mathbb{R}_{+}$and by $L^{2}\left((\lambda \times \nu)^{n}\right)$ the space of square integrable deterministic functions $f:\left(\mathbb{R}_{+} \times \mathbb{R}\right)^{n} \longrightarrow \mathbb{R}$ with respect to the measure $(\lambda \times \nu)^{n}$. If $f$ is a real function on $\left(\mathbb{R}_{+} \times \mathbb{R}\right)^{n}$ we define its symmetrization $(f)^{\wedge}$ with respect to the variables

$\left(t_{1}, x_{1}\right), \ldots,\left(t_{n}, x_{n}\right)$ by

$$
(f)^{\wedge}\left(t_{1}, x_{1}, \ldots, t_{n}, x_{n}\right)=\frac{1}{n !} \sum_{\sigma} f\left(t_{\sigma(1)}, x_{\sigma(1)}, \ldots, t_{\sigma(n)}, x_{\sigma(n)}\right)
$$

where the sum is taken over all permutations $\sigma$ of $\{1, \ldots, n\}$. We say $f$ is symmetric if $f=(f)^{\wedge}$ and we denote by $\widehat{L}^{2}\left((\lambda \times \nu)^{n}\right)$ the space of symmetric functions in $L^{2}\left((\lambda \times \nu)^{n}\right)$.

Naturally, for $f \in \widehat{L}^{2}\left((\lambda \times \nu)^{n}\right)$ one has

$$
\begin{aligned}
& \|f\|_{L^{2}\left((\lambda \times \nu)^{n}\right)}^{2} \\
= & n ! \int_{0}^{\infty} \int_{\mathbb{R}} \ldots \int_{0}^{t_{2}} \int_{\mathbb{R}} f^{2}\left(t_{1}, x_{1}, \ldots, t_{n}, x_{n}\right) d t_{1} \nu\left(d x_{1}\right) \ldots d t_{n} \nu\left(d x_{n}\right)
\end{aligned}
$$

If $f \in L^{2}\left((\lambda \times \nu)^{n}\right)$ we introduce its $n$-fold iterated integral by

$$
J_{n}(f)=\int_{0}^{\infty} \int_{\mathbb{R}} \ldots \int_{0}^{t_{2}} \int_{\mathbb{R}} f\left(t_{1}, x_{1}, \ldots, t_{n}, x_{n}\right) \tilde{N}\left(d t_{1}, d x_{1}\right) \ldots \tilde{N}\left(d t_{n}, d x_{n}\right)
$$

If $f \in \widehat{L}^{2}\left((\lambda \times \nu)^{n}\right)$ we define

$$
I_{n}(f)=n ! J_{n}(f)
$$

Then we get for $f \in \widehat{L}^{2}\left((\lambda \times \nu)^{n}\right)$ and $g \in \widehat{L}^{2}\left((\lambda \times \nu)^{m}\right)$ the following orthogonality relation

$$
E\left[I_{n}(f) I_{m}(g)\right]= \begin{cases}0, & n \neq m \\ n !(f, g)_{L^{2}\left((\lambda \times \nu)^{n}\right)}, & n=m\end{cases}
$$

where $(\cdot, \cdot)_{L^{2}\left((\lambda \times \nu)^{n}\right)}$ is the scalar product for $L^{2}\left((\lambda \times \nu)^{n}\right)$.

Then, with the notation above, the following chaos expansion in terms of iterated integrals with respect to $\widetilde{N}(d t, d x)$ holds

Theorem 2.1: Let $F \in L^{2}(\mu)$. Then there exists a unique sequence of functions $f_{m}$ in $\widehat{L}^{2}\left((\lambda \times \nu)^{m}\right), m \geq 1$ such that

$$
F=E[F]+\sum_{m \geq 1} I_{m}\left(f_{m}\right)
$$

Moreover, the following isometry is valid

$$
E\left[F^{2}\right]=(E[F])^{2}+\sum_{m \geq 1} m !\left\|f_{m}\right\|_{L^{2}\left((\lambda \times \nu)^{m}\right)}^{2} .
$$


Example 2.2 Choose $h \in \widehat{L}^{2}(\lambda \times \nu)$ and define $F \in L^{2}(\mu)$ by $F(\omega)=\int_{\mathbb{R}_{+}} \int_{\mathbb{R}} h(s, x) \tilde{N}(d s, d x)$. Then

$$
F(\omega)=I_{1}\left(f_{1}\right)
$$

So in this case $f_{1}(s, x)=h(s, x)$.

In particular,

$$
\widetilde{N}(t, \Lambda)=I_{1}\left(\chi_{[0, t]}(s) \chi_{\Lambda}(x)\right) ; t \geq 0
$$

for Borel sets $\Lambda \subset \mathbb{R} \backslash\{0\}$, whose closure $\bar{\Lambda}$ does not contain 0 .

\section{White noise of Poisson random measures}

In this section we introduce the concept of white noise for Poisson random measures. Our presentation and notation will follow that of [HØUZ] closely, where Gaussian white noise theory is treated. More information about white noise theory can be found in [HKPS], [Ku] and $[\mathrm{O}]$.

\subsection{Wick product for spaces of smooth and generalized random variables}

First, we briefly recall a chaos expansion result for Lévy processes with kernels of polynomial type, presented in [DØP]. We use this expansion to define a Wick product for spaces of smooth and generalized random variables (see $[\mathrm{D} \emptyset \mathrm{P}]$ ).

Adopting the notation of Section 2, we now suppose that our Lévy measure $\nu$ satisfies the condition of [NS], namely that for every $\varepsilon>0$ there exists a $\lambda>0$ such that

$$
\int_{\mathbb{R} \backslash(-\varepsilon, \varepsilon)} \exp (\lambda|x|) \nu(d x)<\infty .
$$

This implies that our Lévy measure has finite moments of all orders $\geq 2$.

Here and in the forthcoming we will make use of multi-indices of arbitrary length. To simplify the notation, we regard multi-indices as elements of the space $\left(I N_{0}^{N}\right)_{c}$ of all sequences $\alpha=\left(\alpha_{1}, \alpha_{2}, \ldots\right)$ with elements $\alpha_{i} \in \mathbb{N}_{0}$ and with compact support, i.e. with only finitely many $\alpha_{i} \neq 0$. We define

$$
\mathcal{J}=\left(N_{0}^{N}\right)_{c}
$$

Further, we set $\operatorname{Index}(\alpha)=\max \left\{i: \alpha_{i} \neq 0\right\}$ and $|\alpha|=\sum_{i} \alpha_{i}$ for $\alpha \in \mathcal{J}$.

For the chaos expansion theorem we need two families of orthogonal polynomials. First let $\left\{\xi_{k}\right\}_{k \geq 1}$ be the complete orthonormal system of $L^{2}\left(\mathbb{R}_{+}\right)$consisting of the Laguerre functions of order $\frac{1}{2}$, i.e.

$$
\xi_{k}(t)=\left(\frac{\Gamma(k)}{\Gamma(k+1 / 2)}\right)^{1 / 2} e^{-t} t^{1 / 4} L_{k-1}^{1 / 2}(t) \chi_{(0, \infty)}(t), k \geq 1,
$$


where $\Gamma$ is the Gamma function and where $L_{k}^{1 / 2}$ are the Laguerre polynomials of order $\frac{1}{2}$, defined by the formula

$$
e^{-t} t^{1 / 2} L_{k}^{1 / 2}(t)=\frac{1}{k !} \frac{d^{k}}{d t^{k}}\left(e^{-t} t^{k+1 / 2}\right), k \geq 0
$$

See e.g. [T] for the definition. Further, let $\left\{l_{m}\right\}_{m \geq 0}$ be the orthogonalization of $\left\{1, x, x^{2}, \ldots\right\}$ with respect to the innerproduct of $L^{2}(\pi)$, where $\pi(d x)=x^{2} \nu(d x)$. Then define the polynomials

$$
p_{m}(x)=\frac{1}{\left\|l_{m-1}\right\|_{L^{2}(\pi)}} x \cdot l_{m-1}(x)
$$

The polynomials $p_{m}$ form a complete orthonormal system in $L^{2}(\nu)$ (see relation (3.13) in Remark 3.2 for $m=1$ ). Next define the map

$$
z: I N \times I N \longrightarrow I N ;(i, j) \vdash j+(i+j-2)(i+j-1) / 2
$$

Note that $z$ is bijective and gives a formula for the "diagonal counting" of the Cartesian product $I N \times I N$.

Further, if $k=z(i, j)$ for $i, j \in \mathbb{N}$, set

$$
\delta_{k}(t, x)=\xi_{i}(t) p_{j}(x)
$$

Now, suppose $\operatorname{Index}(\alpha)=j$ and $|\alpha|=m$ for $\alpha \in \mathcal{J}$ and define the function $\delta^{\otimes \alpha}$ to be

$$
\begin{gathered}
\delta^{\otimes \alpha}\left(\left(t_{1}, x_{1}\right), \ldots,\left(t_{m}, x_{m}\right)\right)= \\
\delta_{1}^{\otimes \alpha_{1}} \otimes \ldots \otimes \delta_{j}^{\otimes \alpha_{j}}\left(\left(t_{1}, x_{1}\right), \ldots,\left(t_{m}, x_{m}\right)\right)=\delta_{1}\left(t_{1}, x_{1}\right) \cdot \ldots \cdot \delta_{1}\left(t_{\alpha_{1}}, x_{\alpha_{1}}\right) \\
\cdot \ldots \cdot \delta_{j}\left(t_{\alpha_{1}+\ldots+\alpha_{j-1}+1}, x_{\alpha_{1}+\ldots+\alpha_{j-1}+1}\right) \cdot \ldots \cdot \delta_{j}\left(t_{m}, x_{m}\right),
\end{gathered}
$$

where the terms with zero-components $\alpha_{i}$ are set equal to 1 in the product $\left(\delta_{i}^{\otimes 0}=1\right)$.

Finally, we define the symmetrized tensor product of the $\delta_{k}$ 's, denoted by $\delta^{\widehat{\otimes} \alpha}$ as

$$
\begin{aligned}
\delta^{\widehat{\otimes} \alpha}\left(\left(t_{1}, x_{1}\right), \ldots,\left(t_{m}, x_{m}\right)\right) & =\left(\delta^{\otimes \alpha}\right)^{\wedge}\left(\left(t_{1}, x_{1}\right), \ldots,\left(t_{m}, x_{m}\right)\right) \\
& =\delta_{1}^{\widehat{\otimes} \alpha_{1}} \widehat{\otimes} \ldots \widehat{\otimes} \delta_{j}^{\widehat{\otimes} \alpha_{j}}\left(\left(t_{1}, x_{1}\right), \ldots,\left(t_{m}, x_{m}\right)\right) .
\end{aligned}
$$

For $\alpha \in \mathcal{J}$ define

$$
K_{\alpha}:=I_{|\alpha|}\left(\delta^{\widehat{\otimes} \alpha}\right)
$$

where we set $I_{0}\left(\delta^{\widehat{\otimes} 0}\right)=1$. In particular, note that if $\alpha=\epsilon^{l}$ with

$$
\epsilon^{l}(j)=\left\{\begin{array}{l}
1 \text { for } j=l \\
0 \text { else }
\end{array}, l \geq 1\right.
$$


we get (see (3.24))

$$
K_{\epsilon^{l}}=I_{1}\left(\delta^{\widehat{\otimes}^{l}}\right)=I_{1}\left(\delta_{l}\right)=I_{1}\left(\xi_{i}(t) p_{j}(x)\right)
$$

if $l=z(i, j)$.

We can now state the chaos representation result in [DØP] for orthonormal $p_{m}$.

Theorem 3.1 The family $\left\{K_{\alpha}\right\}_{\alpha \in \mathcal{J}}$ constitutes an orthogonal basis for $L^{2}(\mu)$ with norm expression

$$
\left\|K_{\alpha}\right\|_{L^{2}(\mu)}^{2}=\alpha !:=\alpha_{1} ! \alpha_{2} ! \ldots
$$

for $\alpha=\left(\alpha_{1}, \alpha_{2}, \ldots\right) \in \mathcal{J}$. Thus every $F \in L^{2}(\mu)$ has the unique representation

$$
F=\sum_{\alpha \in \mathcal{J}} c_{\alpha} K_{\alpha}
$$

where $c_{\alpha} \in \mathbb{R}$ for all $\alpha$ and where we set $c_{0}=E[F]$.

Moreover, we have the isometry

$$
\|F\|_{L^{2}(\mu)}^{2}=\sum_{\alpha \in \mathcal{J}} \alpha ! c_{\alpha}^{2}
$$

Remark 3.2 By Theorem 2.1 any sequence of functions $f_{m} \in \widehat{L}^{2}\left((\lambda \times \nu)^{m}\right), m=$ $0,1,2, \ldots$ such that $\sum_{m \geq 1} m !\left\|f_{m}\right\|_{L^{2}\left((\lambda \times \nu)^{m}\right)}^{2}<\infty$ defines a random variable $F \in L^{2}(\mu)$ by $F=\sum_{m \geq 0} I_{m}\left(f_{m}\right)$, where by convention, $I_{0}\left(f_{0}\right)=f_{0}$ (constant). It is shown in [DØP] that each $f_{m}$ is contained in the closure of the linear span of the orthogonal family $\left\{\delta^{\widehat{\otimes} \alpha}\right\}_{|\alpha|=m}$ in $L^{2}\left((\lambda \times \nu)^{m}\right)$. So we obtain for all $m \geq 1$

$$
f_{m}=\sum_{|\alpha|=m} c_{\alpha} \delta^{\widehat{\otimes} \alpha}
$$

in $L^{2}\left((\lambda \times \nu)^{m}\right)$ for $c_{\alpha} \in \mathbb{R}$. In particular, the linear span of $\left\{\delta^{\widehat{\otimes} \alpha}\right\}_{|\alpha|=1}$ is dense in $L^{2}(\lambda \times \nu)$.

Example 3.3 (i) Choose $f_{1}(s, x)=\chi_{[0, t]}(s) \chi_{\Lambda}(x)$ as in Example 2.2. Because of $(3.9)$ and (3.13) we get then for $F=\widetilde{N}(t, \Lambda)=I_{1}\left(f_{1}\right)$

$$
\widetilde{N}(t, \Lambda)=\sum_{k, m \geq 1} \int_{0}^{t} \int_{\Lambda} \xi_{k}(s) p_{m}(x) \nu(d x) d s \cdot K_{\epsilon^{z(k, m)}} .
$$

(ii) Choose $F(\omega)=\eta(t)=\int_{0}^{t} \int_{\mathbb{R}} x \tilde{N}(d s, d x)=I_{1}\left(\chi_{[0, t]}(s) x\right)$. Then by $(3.9)$

$$
\eta(t)=\sum_{k \geq 1} m \int_{0}^{t} \xi_{k}(s) d s \cdot K_{\epsilon^{z(k, 1)}}
$$

where $m=\|x\|_{L^{2}(\nu)}$. 
We will recall spaces of smooth and generalized random variables, based on the expansion result (Theorem 3.1). See [DØP].

The following spaces of smooth random variables, $\mathcal{G}=\mathcal{G}(\mu)$, and generalized random variables, $\mathcal{G}^{*}=\mathcal{G}^{*}(\mu)$, have been studied in the Gaussian case by Potthoff and Timpel (1995). See [PT] and the references therein. Later the Poissonian case has been treated in [BG] and [HØ2]. The versions of the spaces with respect to the Lévy white noise measure $\mu$ are constructed, analogously.

Definition 3.4 (i) Let $k \in \mathbb{N}_{0}$. We say that $f=\sum_{m \geq 0} I_{m}\left(f_{m}\right) \in L^{2}(\mu)$ belongs to the space $\mathcal{G}_{k}(\mu)$ if

$$
\|f\|_{\mathcal{G}_{k}}^{2}:=\sum_{m \geq 0} m !\left\|f_{m}\right\|_{L^{2}\left((\lambda \times \nu)^{m}\right)}^{2} e^{2 k m}<\infty
$$

We define

$$
\mathcal{G}=\mathcal{G}(\mu)=\bigcap_{k \in N_{0}} \mathcal{G}_{k}(\mu)
$$

and we equip $\mathcal{G}$ with the projective topology.

(ii) We say that a formal expansion

$$
G=\sum_{m \geq 0} I_{m}\left(g_{m}\right)
$$

belongs to the space $\mathcal{G}_{-q}(\mu)\left(q \in N_{0}\right)$ if

$$
\|G\|_{\mathcal{G}_{-q}}^{2}:=\sum_{m \geq 0} m !\left\|g_{m}\right\|_{L^{2}\left((\lambda \times \nu)^{m}\right)}^{2} e^{-2 q m}<\infty .
$$

We define

$$
\mathcal{G}^{*}=\mathcal{G}^{*}(\mu)=\bigcup_{q \in N_{0}} \mathcal{G}_{-q}(\mu)
$$

and we equip $\mathcal{G}^{*}$ with the inductive topology. Note, that $\mathcal{G}^{*}$ is the dual of $\mathcal{G}$, with dual pairing

$$
\langle G, f\rangle=\sum_{m \geq 0} m !\left(f_{m}, g_{m}\right)_{L^{2}\left((\lambda \times \nu)^{m}\right)}
$$

if $G \in \mathcal{G}^{*}$ and $f \in \mathcal{G}$.

Also note that by (3.7) and (3.13) the connection between the expansion

$$
F=\sum_{m \geq 0} I_{m}\left(f_{m}\right)
$$

and the expansion

$$
F=\sum_{\alpha \in \mathcal{J}} c_{\alpha} K_{\alpha}
$$


is given by the representation (3.13). Since this gives

$$
\left\|I_{m}\left(f_{m}\right)\right\|_{L^{2}(\mu)}^{2}=m !\left\|f_{m}\right\|_{L^{2}\left((\lambda \times \nu)^{m}\right)}^{2}=\sum_{|\alpha|=m} \alpha ! c_{\alpha}^{2}
$$

it follows that we can express the $\mathcal{G}_{r}$-norm of $F$ in terms of the chaos expansion $(2.12)$ as follows:

$$
\|F\|_{\mathcal{G}_{r}}^{2}=\sum_{m \geq 0}\left(\sum_{|\alpha|=m} \alpha ! c_{\alpha}^{2}\right) e^{-2 r m}, r \in \mathbb{Z}
$$

We also recall the Lévy versions of the Hida test function space $(\mathcal{S})$ and the Hida stochastic distribution space $(\mathcal{S})^{*}$. For more information about these or related spaces in the Gaussian and Poissonian case we refer to [HKPS] and [HØUZ].

For a formal expansion $f=\sum_{\alpha \in \mathcal{J}} c_{\alpha} K_{\alpha}$ define the norm

$$
\|f\|_{0, k}^{2}:=\sum_{\alpha \in \mathcal{J}} \alpha ! c_{\alpha}^{2}(2 I N)^{k \alpha}
$$

for integers $k$, where $(2 I N)^{k \alpha}=(2 \cdot 1)^{k \alpha_{1}}(2 \cdot 2)^{k \alpha_{2}} \ldots(2 \cdot m)^{k \alpha_{m}}$, if Index $(\alpha)=m$. Let

$$
(\mathcal{S})_{0, k}:=\left\{f:\|f\|_{0, k}<\infty\right\}
$$

and define

$$
(\mathcal{S}):=\bigcap_{k \in N_{0}}(\mathcal{S})_{0, k}
$$

with the projective topology, and

$$
(\mathcal{S})^{*}:=\bigcup_{k \in N_{0}}(\mathcal{S})_{0, k}
$$

with the inductive topology. Then $(\mathcal{S})^{*}$ is the dual of $(\mathcal{S})$, with dual pairing

$$
\langle G, f\rangle=\sum_{\alpha \in \mathcal{J}} a_{\alpha} b_{\alpha} \alpha !
$$

if $G=\sum_{\alpha \in \mathcal{J}} a_{\alpha} K_{\alpha} \in(\mathcal{S})^{*}$ and $f=\sum_{\alpha \in \mathcal{J}} b_{\alpha} K_{\alpha} \in(\mathcal{S})$. Note that

$$
(\mathcal{S}) \subset \mathcal{G} \subset L^{2}(\mu) \subset \mathcal{G}^{*} \subset(\mathcal{S})^{*}
$$

One of the interesting features of $(\mathcal{S})^{*}$ is that it contains the white noise of the compensated Poisson random measure associated with the Lévy process $\eta(t)$.

Definition 3.5 The white noise $\dot{\widetilde{N}}(t, x)$ of the Poisson random measure $\tilde{N}(d t, d x)$ is defined by the following formal expansion

$$
\dot{\widetilde{N}}(t, x)=\sum_{k, m \geq 1} \xi_{k}(t) p_{m}(x) \cdot K_{\epsilon^{z(k, m)}}
$$


where $\xi_{k}(t)$ are the Laguerre functions, $p_{m}(x)$ the polynomials from $(3.3), z(i, j)$ the map in (3.4) and where $\epsilon^{l} \in \mathcal{J}$ is defined as in (3.8).

Remark 3.6 (i) Because of the relation (3.14) we can interprete $\dot{\widetilde{N}}(t, x)$ as a RadonNikodym derivative, i.e.(formally)

$$
\dot{\widetilde{N}}(t, x)=\frac{\widetilde{N}(d t, d x)}{d t \times \nu(d x)} \text { in }(\mathcal{S})^{*} .
$$

This gives the justification of the name white noise for $\dot{\widetilde{N}}(t, x)$.

(ii) Since $\delta^{\widehat{\otimes} \epsilon^{z(k, m)}}(t, x)=\xi_{k}(s) p_{m}(x)$ and so

$$
\int_{\mathbb{R}_{+}} \int_{\mathbb{R}}\|\dot{\widetilde{N}}(t, x)\|_{0,-q}^{2} \nu(d x) d t=\sum_{n \geq 1} \frac{1}{n^{q}}<\infty, q \geq 2,
$$

the white noise $\dot{\widetilde{N}}(t, x)$ takes values in $(\mathcal{S})^{*} \lambda \times \nu$-a.e. However, $\mathcal{G}^{*}$ is not rich enough to accommodate $\dot{\widetilde{N}}(t, x)$.

(iii) The white noise $\dot{\eta}(t)$ of the Lévy process $\eta(t)$ (with $m=\|x\|_{L^{2}(\nu)}$ ) is defined in [DØP] as

$$
\dot{\eta}(t)=m \sum_{k \geq 1} \xi_{k}(t) K_{\epsilon^{z(k, 1)}}
$$

for all $t \geq 0$. Thus by (3.15) we get that

$$
\dot{\eta}(t)=\frac{d}{d t} \eta(t) \text { in }(\mathcal{S})^{*} \quad(\text { see }(4.12))
$$

Moreover, $\dot{\eta}(t)$ is related to $\dot{\widetilde{N}}(t, x)$ by

$$
\dot{\eta}(t)=\int_{\mathbb{R}} x \stackrel{\dot{\widetilde{N}}}{ }(t, x) \nu(d x) .
$$

This relation, which involves a Bochner integral with respect to $\nu$, can be seen from the proof of equation (4.14).

\subsubsection{Wick product and Itô/Skorohod integration with respect to compensated Poisson random measure}

In this section we recall a (stochastic) Wick product $\diamond$ for the spaces $\mathcal{G}^{*}$ and $(\mathcal{S})^{*}$, defined in $[\mathrm{D} \varnothing \mathrm{P}]$. Then we will apply this concept of product to introduce a Skorohod integral with respect to Poisson random measures. One of the useful properties of this Wick product is its relation to Itô/Skorohod integration. In short, this relation can be expressed as

$$
\int_{\mathbb{R}_{+}} \int_{\mathbb{R}} Y(t, x) \tilde{N}(\delta t, d x)=\int_{\mathbb{R}_{+}} \int_{\mathbb{R}} Y(t, x) \diamond \stackrel{\dot{\tilde{N}}}{(t, x) \nu}(d x) d t .
$$


The left hand side denotes the Skorohod integral of a random field $Y: \mathbb{R}_{+} \times \mathbb{R} \longrightarrow \mathbb{R}$ with respect to the compensated Poisson random measure (which coincides with the corresponding Itô integral, if $Y(t, x)$ is predictable). The right side is to be interpreted as an $(\mathcal{S})^{*}$-valued Bochner-integral. $\dot{\widetilde{N}}(t, x)$ is the white noise of $\widetilde{N}(d t, d x)$ (Definition 3.5).

Definition 3.7 The Lévy Wick product $F \diamond G$ of two elements

$$
F=\sum_{\alpha \in \mathcal{J}} a_{\alpha} K_{\alpha}, G=\sum_{\beta \in \mathcal{J}} b_{\beta} K_{\beta} \in(\mathcal{S})^{*}
$$

is defined by

$$
F \diamond G=\sum_{\alpha, \beta \in \mathcal{J}} a_{\alpha} b_{\beta} K_{\alpha+\beta}
$$

Remark 3.8 Let $f_{n}=\sum_{|\alpha|=n} c_{\alpha} \delta^{\widehat{\otimes} \alpha} \in \widehat{L}^{2}\left((\lambda \times \nu)^{n}\right)$ and $g_{m}=\sum_{|\beta|=m} b_{\beta} \delta^{\widehat{\otimes} \beta}$

$\in \widehat{L}^{2}\left((\lambda \times \nu)^{m}\right)$. Then we have

$$
f_{n} \widehat{\otimes} g_{m}=\sum_{|\alpha|=n} \sum_{|\beta|=m} c_{\alpha} b_{\beta} \delta^{\widehat{\otimes}(\alpha+\beta)}=\sum_{|\gamma|=n+m} \sum_{\alpha+\beta=\gamma} c_{\alpha} b_{\beta} \delta^{\widehat{\otimes} \gamma}
$$

in $L^{2}\left((\lambda \times \nu)^{n+m}\right)$. Therefore we obtain

$$
I_{n}\left(f_{n}\right) \diamond I_{m}\left(g_{m}\right)=I_{n+m}\left(f_{n} \widehat{\otimes} g_{m}\right) .
$$

It is important to note that the spaces $(\mathcal{S}), \mathcal{G}, \mathcal{G}^{*}$ and $(\mathcal{S})^{*}$ form topological algebras with respect to the Lévy Wick product $\diamond$ (for an analogous proof see [PT] and [HØUZ]). For more information about the Wick product and Skorohod integration in the Poissonian and Gaussian case see e.g. [HKPS] ,[HØUZ] and [NZ].

Next, we define a Skorohod integral with respect to $\widetilde{N}(d t, d x)$ in a way, suggested in [NV]. See also $[\mathrm{BL}]$.

Let $Y: \mathbb{R}_{+} \times \mathbb{R} \longrightarrow \mathbb{R}$ be a random field such that

$$
E\left[Y(t, x)^{2}\right]<\infty \text { for all } t, x
$$

Then, by Theorem 2.1, $Y(t, x)$ has a chaos expansion

$$
Y(t, x)=\sum_{m \geq 0} I_{m}\left(f_{m}\left(t_{1}, x_{1}, \ldots, t_{m}, x_{m}, t, x\right)\right)
$$

where $f_{m}(\cdot, t, x) \in \widehat{L}^{2}\left((\lambda \times \nu)^{m}\right)$ for $m \geq 1, t \geq 0, x \in \mathbb{R}$ and where $I_{0}\left(f_{0}\right):=E[Y(t, x)]$. Let $\widehat{f}_{m}\left(t_{1}, x_{1}, \ldots, t_{m+1}, x_{m+1}\right)$ be the symmetrization of

$$
f_{m}\left(t_{1}, x_{1}, \ldots, t_{m}, x_{m}, t_{m+1}, x_{m+1}\right)
$$

with respect to the $m+1$ variables $\left(t_{1}, x_{1}\right), \ldots,\left(t_{m+1}, x_{m+1}\right)$. 
Definition 3.9 If

$$
\sum_{m \geq 0}(m+1) !\left\|\widehat{f}_{m}\right\|_{L^{2}\left((\lambda \times \nu)^{m+1}\right)}^{2}<\infty
$$

then define the Skorohod integral with respect to $\widetilde{N}(d t, d x)$, indicated by

$$
\int_{\mathbb{R}_{+}} \int_{\mathbb{R}} Y(t, x) \tilde{N}(\delta t, d x)
$$

by

$$
\int_{\mathbb{R}_{+}} \int_{\mathbb{R}} Y(t, x) \tilde{N}(\delta t, d x):=\sum_{m \geq 0} I_{m+1}\left(\widehat{f}_{m}\left(t_{1}, x_{1}, \ldots, t_{m+1}, x_{m+1}\right)\right) .
$$

By (3.35) and (3.36) the Skorohod integral belongs to $L^{2}(\mu)$ and

$$
\left\|\int_{\mathbb{R}_{+}} \int_{\mathbb{R}} Y(t, x) \tilde{N}(\delta t, d x)\right\|_{L^{2}(\mu)}^{2}=\sum_{m \geq 0}(m+1) !\left\|\widehat{f}_{m}\right\|_{L^{2}\left((\lambda \times \nu)^{m+1}\right)}^{2} .
$$

Note, that the the process $Y(t, x)$ is not required to be predictable. The next result shows that the Skorohod integral (3.36) is an extension of the Itô integral to non-predictable integrands.

Proposition 3.10 Suppose $Y \in L^{2}\left(\mathbb{R}_{+} \times \mathbb{R} \times \Omega\right)$ is predictable. Then $Y$ is both Skorohod integrable and Itô integrable, and the two integrals coincide:

$$
\int_{\mathbb{R}_{+}} \int_{\mathbb{R}^{R}} Y(t, x) \tilde{N}(\delta t, d x)=\int_{\mathbb{R}_{+}} \int_{\mathbb{R}} Y(t, x) \tilde{N}(d t, d x) .
$$

Proof The proof is based on the same principles as in [DØP, Proposition 3.16]

We wish to prove the integral relation (3.30). For this purpose we define a certain Bochner integral in $(\mathcal{S})^{*}$.

Definition 3.11 We say a generalized random field $Z: R_{+} \times \mathbb{R} \longrightarrow(\mathcal{S})^{*}$ is $(\mathcal{S})^{*}$-integrable if

$$
\langle Z(\cdot), f\rangle \in L^{1}(\lambda \times \nu) \text { for all } f \in(\mathcal{S})
$$

where the dual pairing $\langle\cdot, \cdot\rangle$ is defined in $(3.24)$. Then the $(\mathcal{S})^{*}$-integral of $Z$, denoted by $\int_{\mathbb{R}_{+}} \int_{\mathbb{R}} Z(t, x) \nu(d x) d t$, is the unique element in $(\mathcal{S})^{*}$ such that

$$
\left\langle\int_{\mathbb{R}_{+}} \int_{\mathbb{R}} Z(t, x) \nu(d x) d t, f\right\rangle=\int_{\mathbb{R}_{+}} \int_{\mathbb{R}}\langle Z(t, x), f\rangle \nu(d x) d t
$$

for all $f \in(\mathcal{S})$. 
It follows from Proposition 8.1 in [HKPS] that (3.39) defines $\int_{\mathbb{R}_{+}} \int_{\mathbb{R}} Z(t, x) \nu(d x) d t$ as an element in $(\mathcal{S})^{*}$.

We need the following three Lemmas:

Lemma 3.12 Let $Z(t, x)=\sum_{\alpha \in \mathcal{J}} c_{\alpha}(t, x) K_{\alpha} \in(\mathcal{S})^{*}$ for all $t \geq 0, x \in \mathbb{R}$ with

$$
\sum_{\alpha \in \mathcal{J}} \alpha !\left\|c_{\alpha}\right\|_{L^{1}\left(\mathbb{R}_{+} \times \mathbb{R}\right)}^{2}(2 N)^{-p \alpha}<\infty
$$

for some $p<\infty$. Then $Z$ is $(\mathcal{S})^{*}$-integrable and

$$
\int_{\mathbb{R}_{+}} \int_{\mathbb{R}} Z(t, x) \nu(d x) d t=\sum_{\alpha} \int_{\mathbb{R}_{+}} \int_{\mathbb{R}} c_{\alpha}(t, x) \nu(d x) d t K_{\alpha}
$$

Proof Analogous to the proof of Lemma 2.5.6 in [HØUZ].

Lemma 3.13 Assume $Y(t, x)=\sum_{\alpha \in \mathcal{J}} c_{\alpha}(t, x) K_{\alpha} \in(\mathcal{S})^{*}$ for all $t, x$ with

$$
L:=\sup _{\alpha}\left\{\alpha !\left\|c_{\alpha}\right\|_{L^{2}\left(\mathbb{R}_{+} \times \mathbb{R}\right)}^{2}(2 I N)^{-q \alpha}\right\}<\infty
$$

for some $q<\infty$. Then $Y(t, x) \diamond \dot{\widetilde{N}}(t, x)$ is $(\mathcal{S})^{*}$-integrable and

$$
\int_{\mathbb{R}_{+}} \int_{\mathbb{R}} Y(t, x) \diamond \dot{\widetilde{N}}(t, x) \nu(d x) d t=\sum_{\alpha, k, m} \int_{\mathbb{R}_{+}} \int_{\mathbb{R}} c_{\alpha}(t, x) \xi_{k}(t) p_{m}(x) \nu(d x) d t K_{\alpha+\epsilon^{z(k, 1)}}
$$

with the map $z(i, j)$ as in (3.4) and $\epsilon^{l}$ as in (3.8).

Proof The definition of the Wick product (Definition 3.7) supplies

$$
Y(t, x) \diamond \dot{\widetilde{N}}(t, x)=\sum_{\beta} \sum_{\substack{\alpha, k, m: \\ \alpha+\epsilon^{z(k, m)}=\beta}} c_{\alpha}(t, x) \xi_{k}(t) p_{m}(x) K_{\beta}
$$

Because of Lemma 3.12 we have to verify that

$$
M(\beta):=\sum_{\beta} \beta !\left\|\sum_{\substack{\alpha, k, m: \\ \alpha+\epsilon^{z(k, m)}=\beta}} c_{\alpha}(t, x) \xi_{k}(t) p_{m}(x)\right\|_{L^{1}\left(\mathbb{R}_{+} \times \mathbb{R}_{+}\right)}^{2}(2 N)^{-p \alpha}<\infty
$$

for some $p<\infty$. It follows then

$$
\int_{\mathbb{R}_{+}} \int_{\mathbb{R}^{R}}\left|c_{\alpha}(t)\right|\left|\xi_{k}(t) p_{m}(x)\right| \nu(d x) d t \leq\left\|c_{\alpha}\right\|_{L^{2}\left(\mathbb{R}_{+} \times \mathbb{R}\right)}
$$

Further, we get

$$
\begin{aligned}
& \left\|\sum_{\substack{\alpha, k, m: \\
\alpha+\epsilon^{z k, m)}=\beta}} c_{\alpha}(t, x) \xi_{k}(t) p_{m}(x)\right\|_{L^{2}\left(\mathbb{R}_{+} \times \mathbb{R}\right)}^{2} \leq\left[\sum_{\alpha, \gamma: \alpha+\gamma=\beta}\left\|c_{\alpha}\right\|_{L^{2}\left(\mathbb{R}_{+} \times \mathbb{R}\right)} \chi_{\left\{\epsilon^{k}: k \geq 1\right\}}(\gamma)\right]^{2} \\
& \leq \quad(\operatorname{Index}(\beta))^{2} \sum_{\alpha, \gamma: \alpha+\gamma=\beta}\left\|c_{\alpha}\right\|_{L^{2}\left(\mathbb{R}_{+} \times \mathbb{R}\right)}^{2} \chi_{\left\{\epsilon^{k}: k \geq 1\right\}}(\gamma)
\end{aligned}
$$


This yields the estimate

$$
\begin{aligned}
M(2 q) & \leq \sum_{\alpha, k}\left(\alpha+\epsilon^{k}\right) !\left(\operatorname{Index}\left(\alpha+\epsilon^{k}\right)\right)^{2}\left\|c_{\alpha}\right\|_{L^{2}\left(\mathbb{R}_{+} \times \mathbb{R}\right)}^{2}(2 N)^{-2 q\left(\alpha+\epsilon^{k}\right)} \\
& \leq L \sum_{\alpha, k} \frac{\left(\alpha+\epsilon^{k}\right) !}{\alpha !}\left(\operatorname{Index}\left(\alpha+\epsilon^{k}\right)\right)^{2}(2 \mathbb{I})^{-q \alpha}(2 \mathbb{I})^{-2 q \epsilon^{k}}
\end{aligned}
$$

Further, we observe that

$$
\operatorname{Index}(\gamma) \leq(2 I N)^{\gamma}
$$

This leads finally to

$$
\begin{aligned}
& M(2 q) \leq L \sum_{\alpha, k}(|\alpha|+1)(2 I N)^{2\left(\alpha+\epsilon^{k}\right)}(2 I N)^{-q \alpha}(2 I N)^{-2 q \epsilon^{k}} \\
\leq & L \sum_{k}(2 I N)^{-(2 q-2) \epsilon^{k}} \sum_{\alpha}(|\alpha|+1) 2^{-|\alpha|}(2 I N)^{-(q-3) \alpha}<\infty
\end{aligned}
$$

for $q>4$. The proof follows.

Remark 3.14 One sees easily that the assumptions of the last Lemma are fulfilled if

$$
\int_{\mathbb{R}_{+}} \int_{\mathbb{R}} E\left[Y(t, x)^{2}\right] \nu(d x) d t<\infty .
$$

Lemma 3.15 Suppose $Y(t, x)=\sum_{\alpha \in \mathcal{J}} c_{\alpha}(t, x) K_{\alpha}$ is Skorohod integrable and satisfies the integrability condition (3.40). Then

$$
\int_{\mathbb{R}_{+}} \int_{\mathbb{R}} Y(t, x) \tilde{N}(\delta t, d x)=\sum_{\alpha} \sum_{k, m}\left(c_{\alpha}, \xi_{k} p_{m}\right)_{L^{2}\left(\mathbb{R}_{+} \times \mathbb{R}\right)} K_{\alpha+\epsilon^{z(k, m)}}
$$

Proof $Y(t, x)$ has the chaos expansion

$$
Y(t, x)=\sum_{m \geq 0} I_{m}\left(f_{m}\left(t_{1}, x_{1}, \ldots, t_{m}, x_{m}, t, x\right)\right)
$$

where $f_{m}(\cdot, t, x) \in \widehat{L}^{2}\left((\lambda \times \nu)^{m}\right)$ for $m \geq 1$. Then, by definition of the Skorohod integral and relation (3.13), we get

$$
\begin{aligned}
& \int_{\mathbb{R}_{+}} \int_{\mathbb{R}} Y(t, x) \tilde{N}(\delta t, d x)=\sum_{m \geq 0} I_{m+1}\left(\left(f_{m}\left(t_{1}, x_{1}, \ldots, t_{m}, x_{m}, t, x\right)\right)^{\wedge}\right) \\
= & \sum_{m \geq 0} I_{m+1}\left(\sum_{|\alpha|=m}\left(c_{\alpha}(t, x) \cdot \delta^{\widehat{\otimes} \alpha}\left(t_{1}, x_{1}, \ldots, t_{m}, x_{m}\right)\right)^{\wedge}\right) \\
= & \sum_{m \geq 0} \sum_{|\alpha|=m} \sum_{k, l}\left(c_{\alpha}, \xi_{k} p_{l}\right)_{L^{2}\left(\mathbb{R}_{+} \times \mathbb{R}\right)} I_{m+1}\left(\left(\xi_{k}(t) p_{l}(x) \delta^{\widehat{\otimes} \alpha}\left(t_{1}, x_{1}, \ldots, t_{m}, x_{m}\right)\right)^{\wedge}\right) \\
= & \sum_{m \geq 0} \sum_{|\alpha|=m} \sum_{k, l}\left(c_{\alpha}, \xi_{k} p_{l}\right)_{L^{2}\left(\mathbb{R}_{+} \times \mathbb{R}\right)} I_{m+1}\left(\delta^{\widehat{\otimes} \alpha+\epsilon^{z(k, l)}}\right) \\
= & \sum_{\alpha} \sum_{k, l}\left(c_{\alpha}, \xi_{k} p_{l}\right)_{L^{2}\left(\mathbb{R}_{+} \times \mathbb{R}\right)} K_{\alpha+\epsilon^{z(k, l)}} .
\end{aligned}
$$


We are coming to the main result of this section.

Theorem 3.16 Assume that $Y(t, x)=\sum_{\alpha \in \mathcal{J}} c_{\alpha}(t, x) K_{\alpha}$ is a Skorohod integrable stochas-

tic process with $\int_{a}^{b} \int_{\mathbb{R}} E\left[Y(t, x)^{2}\right] \nu(d x) d t<\infty$ for some $0 \leq a<b$. Then $Y(t, x) \diamond \dot{\widetilde{N}}(t, x)$ is $(\mathcal{S})^{*}$-integrable over $[a, b] \times \mathbb{R}$ and we obtain

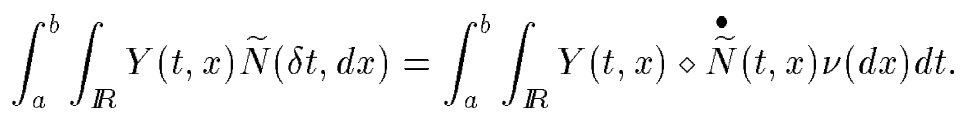

Proof Apply Remark 3.14 and Lemma 3.15 to the process $\chi_{[a, b]}(t) Y(t, x)$.

Remark 3.17 Several parts of the theory can be carried out without the explicit use of the basis $\left\{\xi_{k}(t) p_{m}(x)\right\}_{k, m \geq 1}$. In some cases, however, the choice of an explicit basis provides more information and can be used for example to derive relevant estimates of $\xi_{k}(t) p_{m}(x)$ (see e.g. the proof of relation (4.14)).

\section{Application: A generalized Clark-Haussmann-Ocone theo- rem for Lévy processes}

We now use the framework of the last section to prove a white noise generalization of the Clark-Haussmann-Ocone theorem for Lévy processes. The classical Clark-Haussmann-Ocone (CHO) theorem states that if $F=F(\omega) \in L^{2}(P)$ is $\mathcal{F}_{T}$-measurable and $F \in \mathbb{D}_{1,2}$, then

$$
F(\omega)=E[F]+\int_{0}^{T} E\left[D_{t} F \mid \mathcal{F}_{t}\right] d B(t, \omega)
$$

where $D_{t}$ denotes the Malliavin derivative with domain $D_{1,2}$ and where $B(t, \omega)$ is a Brownian motion on a probability space $(\Omega, \mathcal{F}, P)$ with filtration $\mathcal{F}_{t}$, generated by $B(t, \omega)$. This result and its generalizations have important applications in finance, where $E\left[D_{t} F \mid \mathcal{F}_{t}\right]$ can be regarded as the replicating portfolio of a given $T$-claim $F$ (see e.g. [KO], [Ø]).

Usually this result is presented and proved in the context of analysis on the Wiener space $\Omega=C_{0}([0, T]$ ), the space of all real continuous functions on $[0, T]$ (see e.g. $[\mathrm{N}],[\mathrm{M}],[\ddot{\mathrm{U}} 2]$ ). The disadvantage of this setting is that one has to verify that $F$ is contained in the domain $\mathbb{I}_{1,2}$. This excludes interesting applications. For example, the digital option of the form

$$
F(\omega)=\chi_{[K, \infty)}\left(B_{T}(\omega)\right)
$$

is not in $\mathbb{D}_{1,2}$ and (4.1) cannot be applied to compute the hedging portfolio of the $T$-claim $F$. Recently, (4.1) was generalized to a CHO formula in the setting of white noise analysis (see [AØPU]). This CHO theorem is valid for all $\mathcal{F}_{T}$-measurable $F \in \mathcal{G}^{*}\left(\mu_{0}\right)$, a space of stochastic distributions, similar to that in Definition 3.4, which contains $L^{2}\left(\mu_{0}\right)$, where $\mu_{0}$ is the white noise probability measure with respect to the Brownian motion. The generalization has the form

$$
F=E[F]+\int_{0}^{T} E\left[D_{t} F \mid \mathcal{F}_{t}\right] \hat{\diamond} W_{t} d t
$$


where $\hat{\diamond}$ denotes a Wick product and $W_{t}$ is the white noise of $B_{t}$. Moreover, $E[F]$ is a generalized expectation of $F \in \mathcal{G}^{*}\left(\mu_{0}\right)$ and $E\left[D_{t} F \mid \mathcal{F}_{t}\right]$ is a generalized conditional expectation. The integral on the right hand side is a Bochner integral as in (3.39). In the Wiener space setting another generalization of (4.1) for Meyer-Watanabe distributions $F \in \mathbb{D}-\infty_{-\infty} \varsubsetneqq \mathcal{G}^{*}\left(\mu_{0}\right)$ has

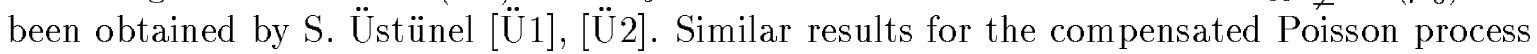
can be found e.g. in [AØPU] and [BH].

We want to prove a corresponding version of (4.2) for Lévy processes. Financial markets modelled by a Brownian motion or a Poisson process are complete. In this case the CHO theorem gives an almost direct formula for the replicating portfolio of a contingent claim in terms of the conditional expectation of its Malliavin derivative. However, financial markets modelled by Lévy processes are in general not complete and, as we will see, the CHO formula does not admit a similar interpretation. On the other hand, it can be used to obtain an explicit formula for the closest hedge, in the sense of minimal variance. See [BDLØP] and Remark 4.4 below.

Let us recall the definition of the following Malliavin derivative $D_{t, x}$, studied in [L]. See also $[\mathrm{NV}]$.

Definition 4.1 Fix a $T>0$. Define $\mathbb{D}_{1,2}$ to be the set of all $\mathcal{F}_{T}$-measurable $F \in L^{2}(\mu)$ with chaos expansion (3.2), i.e.

$$
F=E[F]+\sum_{n \geq 1} I_{n}\left(f_{n}\right)
$$

such that

$$
\|F\|_{\mathbb{D}_{1,2}}^{2}:=\sum_{n \geq 1} n \cdot n !\left\|f_{n}\right\|_{L^{2}\left((\lambda \times \nu)^{n}\right)}^{2}<\infty
$$

The stochastic derivative $D_{t, x}: D_{1,2} \longrightarrow L^{2}(\Omega \times[0, T] \times \mathbb{R}, \mu \times \lambda \times \nu)$ is defined by

$$
D_{t, x} F:=\sum_{n \geq 1} n I_{n-1}\left(f_{n}(\cdot, t, x)\right)
$$

where $f_{n}(\cdot, t, x)=f_{n}\left(t_{1}, x_{1}, \ldots, t_{n-1}, x_{n-1}, t, x\right)$.

Remark 4.2 It is proven in [L] that $D_{t, x}$ is a closed operator and that it coincides with a certain difference operator, defined by J. Picard [Pi].

It is well-known (see [JS]) that every $\mathcal{F}_{T}$-measurable stochastic variable in $L^{2}(\mu)$ can be represented as $F=E[F]+\int_{0}^{T} \int_{\mathbb{R}} \varphi(t, x) \tilde{N}(d t, d x)$ for predictable $\varphi$. If $F \in \mathbb{D}_{1,2}$ then $\varphi$ can be expressed in terms of the operator $D_{t, x}$.

Theorem 4.3 ([L])(CHO theorem for Lévy processes)

Let $F \in \mathbb{D}_{1,2}$ then

$$
F=E[F]+\int_{0}^{T} \int_{\mathbb{R}} E\left[D_{t, x} F \mid \mathcal{F}_{t}\right] \tilde{N}(d t, d x)
$$


Remark 4.4 Let us consider a financial market, consisting of a bond $S_{0} \equiv 1$ and a risky asset $S_{1}$, modelled by the Lévy process $\eta(t)$. It is shown in [BDLØP] that the strategy

$$
\pi_{t}=\frac{1}{\|x\|_{L^{2}(\nu)}^{2}} \int_{\mathbb{R}} x E\left[D_{t, x} F \mid \mathcal{F}_{t}\right] \nu(d x)
$$

realizes the "closest" hedge to the claim $F \in D_{1,2}$ in the sense of minimal variance, i.e. in the sense that the square mean distance between the hedged wealth and the pay off $F$ is minimal. This problem has been studied by several other authors (see e.g. [S], [FSo]). If $\eta(t)$ is a compensated Poisson process then the claim $F$ is replicable.

Remark 4.5 Similarly to the Brownian motion case Theorem 4.3 does not cover the following important example of a claim: The digital option of the form

$$
F(\omega)=\chi_{[K, \infty)}\left(\eta_{T}(\omega)\right)
$$

is not contained in $D_{1,2}$, in general. Take for example a driftless pure jump Lévy process $\eta(t)$ with Lévy measure $\nu(d x)=\chi_{(0,1)}(x) \frac{1}{x^{2}} d x$. So $\eta(t)$ is of unbounded variation, satisfying the integrability condition (3.1). We get then for the real part of its characteristic exponent $\Psi$ that $\operatorname{Re} \Psi(\lambda)=|\lambda| \int_{0}^{|\lambda|}(1-\cos x) \frac{1}{x^{2}} d x$. This yields $\lim _{|\lambda| \rightarrow \infty}|\lambda|^{-\frac{1}{2}} \operatorname{Re} \Psi(\lambda)=\infty$, so that the semigroup of $\eta(t)$ has the strong Feller property. The latter is equivalent to the law of $\eta(T)$ being absolutely continuous with respect to the Lebesgue measure (see [B]), i.e. we have $\mu(\eta(T) \leq K)=\int_{(-\infty, K]} f d t$ for some integrable $f$. So there exists a $K_{0}>0$ such that $f\left(K_{0}\right)>0$ and $\mu(\eta(T) \leq K)$ is differentiable in $K_{0}$. Then it is not difficult to see that $\rho:=\inf _{x \in\left(0, K_{0} / 2\right]} \frac{\mu\left(K_{0}-x \leq \eta(T)<K_{0}\right)}{|x|}>0$. This and the unbounded variation of $\eta(t)$ imply

$$
\int_{\mathbb{R}} \mu\left(K_{0}-x \leq \eta(T)<K_{0}\right) \nu(d x) \geq \int_{\left(-\infty, \frac{K_{0}}{2}\right]} \rho|x| \nu(d x)=\infty .
$$

Suppose $F \in D_{1,2}$, then Lemma 9 in [L] gives

$$
\begin{aligned}
& \int_{0}^{T} \int_{\mathbb{R}} E\left[\left(D_{t, x} F\right)^{2}\right] \nu(d x) d x \\
= & \int_{0}^{T} \int_{\mathbb{R}} E\left[\left(\chi_{[K, \infty)}\left(\eta_{T}(\omega)+x\right)-\chi_{[K, \infty)}\left(\eta_{T}(\omega)\right)\right)^{2}\right] \nu(d x) d x \\
= & T \int_{\mathbb{R}} \mu\left(K_{0}-x \leq \eta(T)<K_{0}\right) \nu(d x)<\infty
\end{aligned}
$$

But this leads to a contradiction. Thus $F \notin \mathbb{D}_{1,2}$.

Remark 4.6 Léon et al. [LSUV] proved a different CHO theorem, based on a chaos expansion of Nualart and Schoutens [NS]. The theorem is in terms of stochastic derivatives $D_{t}^{(m)}: \mathbf{D}_{1,2} \longrightarrow L^{2}(\Omega \times[0, T]), m \geq 1$ and it has the form

$$
F=E[F]+\sum_{m \geq 1} \int_{0}^{T} E\left[D_{t}^{(m)} F \mid \mathcal{F}_{t}\right] d Y_{t}^{(m)}
$$


where $F \in \mathbf{D}_{1,2}$ and where $Y_{t}^{(m)}, m \geq 1$ are power jump processes. The operator $D_{t, x}$ in Definition 4.1 is related to the operators $D_{t}^{(m)}$ as follows

$$
D_{t, x} F=\sum_{m \geq 1}\left\|p_{m}\right\|_{L^{2}(\nu)} p_{m}(x) D_{t}^{(m)} F
$$

$\lambda \times \nu$-a.e. for $F \in D_{1,2} \cap \mathbf{D}_{1,2}$. This follows easily from the definition of the stochastic derivatives.

We need the following definition of conditional expectation in the space $\mathcal{G}^{*}$ (see [DØP]).

Definition 4.7 Let $F=\sum_{m \geq 0} I_{m}\left(f_{m}\right) \in \mathcal{G}^{*}$. Then the conditional expectation of $F$ with respect to $\mathcal{F}_{t}$ is defined by

$$
E\left[F \mid \mathcal{F}_{t}\right]=\sum_{m \geq 0} I_{m}\left(f_{m} \cdot \chi_{[0, t]^{m}}\right)
$$

Note that this coincides with the usual conditional expectation if $F \in L^{2}(\mu)$. Obviously, we get

$$
\left\|E\left[F \mid \mathcal{F}_{t}\right]\right\|_{\mathcal{G}_{r}} \leq\|F\|_{\mathcal{G}_{r}}
$$

for all $r \in \mathbb{Z}$. Thus

$$
E\left[F \mid \mathcal{F}_{t}\right] \in \mathcal{G}^{*}
$$

for all $t$.

Lemma 4.8 For $F, G \in \mathcal{G}^{*}$ we have

$$
E\left[F \diamond G \mid \mathcal{F}_{t}\right]=E\left[F \mid \mathcal{F}_{t}\right] \diamond E\left[G \mid \mathcal{F}_{t}\right]
$$

Proof See $[\mathrm{D} \emptyset \mathrm{P}]$.

Definition 4.1 gives rise to the following generalization of the stochastic derivative $D_{t, x}$ to $\mathcal{G}^{*}$.

Definition 4.9 Let $F=\sum_{\alpha} c_{\alpha} K_{\alpha} \in \mathcal{G}^{*}$. Then define the stochastic derivative of $F$ at $(t, x)$ by

$$
\begin{aligned}
D_{t, x} F & :=\sum_{\alpha} c_{\alpha} \sum_{i} \alpha_{i} K_{\alpha-\epsilon^{i}} \cdot \delta^{\widehat{\otimes} \epsilon^{i}}(t, x) \\
& =\sum_{\alpha} c_{\alpha} \sum_{k, m} \alpha_{z(k, m)} K_{\alpha-\epsilon^{z(k, m)}} \cdot \xi_{k}(t) p_{m}(x) \\
& =\sum_{\beta}\left(\sum_{k, m} c_{\beta+\epsilon^{z(k, m)}}\left(\beta_{z(k, m)}+1\right) \xi_{k}(t) p_{m}(x)\right) K_{\beta},
\end{aligned}
$$


with the map $z(i, j)$ in (3.4) and $\epsilon^{l}$ as in (3.8).

Example 4.10 Let $X=\int_{0}^{\infty} \int_{\mathbb{R}} \xi_{k}(t) p_{m}(x) \tilde{N}(d t, d x)$ for some $k, m \geq 1$. Then $(4.10)$ gives $D_{t, x} X=\xi_{k}(t) p_{m}(x)$.

We need the following result.

\section{Lemma 4.11}

(i) Let $F \in \mathcal{G}^{*}$. Then $D_{t, x} F \in \mathcal{G}^{*} \lambda \times \nu$-a.e.

(ii) Suppose $F, F_{n} \in \mathcal{G}^{*}$ for all $n \in \mathbb{I N}$ and

$$
F_{n} \longrightarrow F \text { in } \mathcal{G}^{*}
$$

Then there exists a subsequence $\left\{F_{n_{k}}\right\}_{k \geq 1}$ such that

$$
D_{t, x} F_{n_{k}} \longrightarrow D_{t, x} F
$$

in $\mathcal{G}^{*} \lambda \times \nu-$ a.e.

Proof It can be shown in same way as in [AØPU, Lemma 3.8] that for all $F \in \mathcal{G}^{*}$ there exists a $p<\infty$ such that

$$
\int_{\mathbb{R}_{+}} \int_{\mathbb{R}}\left\|D_{t, x} F\right\|_{\mathcal{G}_{-p}}^{2} \nu(d x) d t \leq \text { const. } \cdot\|F\|_{\mathcal{G}_{-p+1}}^{2} .
$$

The proof follows then immediately

Remark 4.12 The definition of the derivative $D_{t, x} F$ coincides with the previous one in Definition 4.1, if $F \in \mathbb{D}_{1,2} \subset \mathcal{G}^{*}$. This follows with the help of the closability of the operators and a duality formula for the operators (see [BL] in the case of Definition 4.1). A duality or integration by parts formula for $D_{t, x}$ in Definition 4.9 can be stated as

$$
\int_{\mathbb{R}_{+}} \int_{\mathbb{R}^{R}}<Y(t, x), D_{t, x} F>\nu(d x) d t=<\int_{\mathbb{R}_{+}} \int_{\mathbb{R}} Y(t, x) \diamond \stackrel{\tilde{N}}{ }(t, x) \nu(d x) d t, F>
$$

where $F \in(\mathcal{S})$ and $\int_{\mathbb{R}_{+}} \int_{\mathbb{R}}\|Y(t, x)\|_{\mathcal{G}_{-q}}^{2} \nu(d x) d t<\infty$ for some $q \geq 0$. The latter can be easily seen from the definitions and Lemma 3.13.

In the forthcoming, if

$$
P(x)=\sum_{\alpha} c_{\alpha} x^{\alpha} ; x \in \mathbb{R}^{\mathbb{I N}}, c_{\alpha} \in \mathbb{R}
$$

is a polynomial, where $x^{\alpha}:=x_{1}^{\alpha_{1}} x_{2}^{\alpha_{2}} \ldots$ and $x_{j}^{0}:=1$, we define its Wick version at $X=$ $\left(X_{1}, \ldots, X_{m}\right)$ by

$$
P^{\diamond}(X)=\sum_{\alpha} c_{\alpha} X^{\diamond \alpha}
$$

Further, we call a process $X: \mathbb{R}_{+} \longrightarrow(\mathcal{S})^{*}$ differentiable if the limit

$$
\lim _{\Delta t \rightarrow 0} \frac{X(t+\Delta t)-X(t)}{\Delta t} \text { exists in }(\mathcal{S})^{*}
$$


for all $t$. We denote the derivative of $X(t)$ by $\frac{d}{d t} X(t)$. Define

$$
X_{k, m}^{(t)}=\int_{0}^{t} \int_{\mathbb{R}} \xi_{k}(s) p_{m}(x) \tilde{N}(d s, d x), k, m \geq 1 .
$$

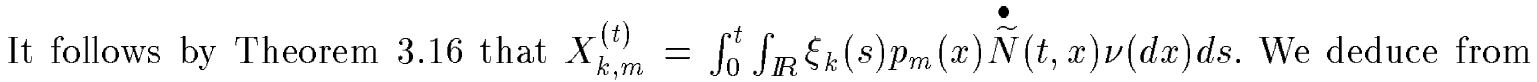
the proof of Lemma 2.8.4 in [HØUZ] that $X_{k, m}^{(t)}$ is differentiable and

$$
\frac{d}{d t} X_{k, m}^{(t)}=\xi_{k}(t) L_{m}(t) \in(\mathcal{S})^{*}
$$

with

$$
L_{m}(t):=\int_{\mathbb{R}} p_{m}(x) \stackrel{\dot{\widetilde{N}}}{(t, x) \nu(d x)}
$$

where we use a Bochner integral with respect to $\nu$, which is analogously defined to Definition 3.11 .

We obtain by induction the following Wick chain rule for polynomials:

Lemma 4.13 Let

$$
P(x)=\sum_{\alpha} c_{\alpha} x^{\alpha}
$$

be a polynomial in $\mathbb{R}^{n}$. Suppose $k_{i}, m_{i} \geq 1$ for all $i=1, \ldots, n$ and let

$$
X^{(t)}=\left(X_{k_{1}, m_{1}}^{(t)}, \ldots, X_{k_{n}, m_{n}}^{(t)}\right)
$$

with $X_{k, m}^{(t)}$ as in (4.13). Then

$$
\frac{d}{d t} P^{\diamond}\left(X^{(t)}\right)=\sum_{i=1}^{n}\left(\frac{\partial P}{\partial x_{i}}\right)^{\diamond}\left(X^{(t)}\right) \diamond L_{m_{i}}(t) \cdot \xi_{k_{i}}(t)
$$

\section{Lemma 4.14 (CHO formula for polynomials)}

Let $F=P^{\diamond}\left(X^{(T)}\right)$ for some polynomial $P(x)=\sum_{\alpha} c_{\alpha} x^{\alpha}$ and the variable $X^{(T)}$ as in Lemma 4.13. Then

$$
F=E[F]+\int_{0}^{T} \int_{\mathbb{R}} E\left[D_{t, x} F \mid \mathcal{F}_{t}\right] \tilde{N}(d t, d x)
$$

Proof Without loss of generality, let us assume that $F=K_{\alpha}^{(T)}$ with $K_{\alpha}^{(t)}:=E\left[K_{\alpha} \mid \mathcal{F}_{t}\right]$ and

$$
\alpha=\alpha_{z\left(k_{1}, m_{1}\right)} \epsilon^{z\left(k_{1}, m_{1}\right)}+\ldots+\alpha_{z\left(k_{n}, m_{n}\right)} \epsilon^{z\left(k_{n}, m_{n}\right)}
$$




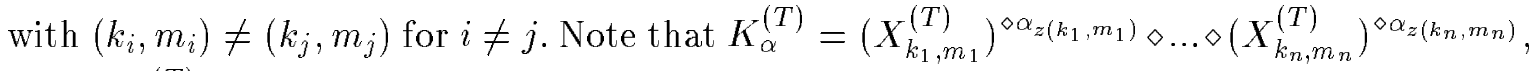
where $X_{k_{i}, m_{i}}^{(T)}$ is as in (4.13).

We conclude from Definition 4.9, Theorem 3.16, a version of Lemma 3.12 for $\nu$ and from Lemma 4.13 that

$$
\begin{aligned}
& \int_{0}^{T} \int_{\mathbb{R}} E\left[D_{t, x} F \mid \mathcal{F}_{t}\right] \tilde{N}(d t, d x) \\
= & \int_{0}^{T} \int_{\mathbb{R}}\left(E\left[\sum_{k, m \geq 1} \alpha_{z(k, m)} K_{\alpha-\epsilon^{z(k, m)}} \cdot \xi_{k}(t) p_{m}(x) \mid \mathcal{F}_{t}\right]\right) \diamond \dot{\widetilde{N}}(t, x) \nu(d x) d t \\
= & \int_{0}^{T} \int_{\mathbb{R}}\left(\sum_{k, m \geq 1} \alpha_{z(k, m)} K_{\alpha-\epsilon^{z(k, m)}}^{(t)} \cdot \xi_{k}(t) p_{m}(x)\right) \diamond \dot{\widetilde{N}}(t, x) \nu(d x) d t \\
= & \int_{0}^{T} \sum_{k, m \geq 1} \alpha_{z(k, m)} K_{\alpha-\epsilon^{z(k, m)}}^{(t)} L_{m}(t) \cdot \xi_{k}(t) d t \\
= & \int_{0}^{T} \frac{d}{d t} K_{\alpha}^{(t)} d t=K_{\alpha}^{(T)}-K_{\alpha}^{(0)}=F-E[F] .
\end{aligned}
$$

We can now prove a CHO theorem for $L^{2}(\mu)$. It is an extension to $L^{2}(\mu)$ of the CHO theorem of $[\mathrm{L}]$ (see Theorem 4.3) and a Poisson random measure version of Theorem 4.13 in [DØP].

Theorem 4.15 (CHO theorem for $L^{2}(\mu)$ )

Let $F \in L^{2}(\mu)$ be $\mathcal{F}_{T}$-measurable. Then

$$
(t, \omega) \mapsto E\left[D_{t, x} F \mid \mathcal{F}_{t}\right] \in L^{2}(\lambda \times \nu \times \mu)
$$

and

$$
F=E[F]+\int_{0}^{T} \int_{\mathbb{R}} E\left[D_{t, x} F \mid \mathcal{F}_{t}\right] \tilde{N}(d t, d x)
$$

Proof Because of Theorem 3.1 there exists a sequence of $\mathcal{F}_{T}$-measurable random variables $F_{n}$ as in the last Lemma such that $F_{n} \longrightarrow F$ in $L^{2}(\mu)$. Then by Lemma 4.14, we get

$$
F_{n}=E\left[F_{n}\right]+\int_{0}^{T} \int_{\mathbb{R}} E\left[D_{t, x} F_{n} \mid \mathcal{F}_{t}\right] \tilde{N}(d t, d x)
$$

for all $n$. By a representation theorem of Itô (see e.g. [JS]) there exists a unique predictable process $u(t, x, \omega)$ such that

$$
E\left[\int_{0}^{T} \int_{\mathbb{R}} u^{2}(t, x, \omega) \nu(d x) d t\right]<\infty
$$


and

$$
F=E[F]+\int_{0}^{T} \int_{\mathbb{R}} u(t, x, \omega) \tilde{N}(d t, d x)
$$

Further we have

$$
\begin{aligned}
& E\left[\int_{0}^{T} \int_{\mathbb{R}}\left(E\left[D_{t, x} F_{n} \mid \mathcal{F}_{t}\right]-u(t, x, \omega)\right)^{2} \nu(d x) d t\right] \\
= & E\left[\left(F_{n}-F-E\left[F_{n}\right]+E[F]\right)^{2}\right] \rightarrow 0 \text { as } n \longrightarrow 0 .
\end{aligned}
$$

So

$$
E\left[D_{t, x} F_{n} \mid \mathcal{F}_{t}\right] \longrightarrow u(t, x, \omega) \text { in } L^{2}(\lambda \times \nu \times \mu)
$$

On the other hand, by taking a subsequence $\left\{F_{n_{k}}\right\}_{k \geq 1}$, we know from Lemma 4.12 that

$$
E\left[D_{t, x} F_{n_{k}} \mid \mathcal{F}_{t}\right] \longrightarrow E\left[D_{t, x} F \mid \mathcal{F}_{t}\right] \text { in } \mathcal{G}^{*} \lambda \times \nu-\text { a.e. }
$$

Taking again a subsequence, we have that

$$
E\left[D_{t, x} F_{n_{k}} \mid \mathcal{F}_{t}\right] \longrightarrow u(t, x, \omega) \text { in } L^{2}(\mu) \lambda \times \nu-\text { a.e. }
$$

It follows

$$
F=E[F]+\int_{0}^{T} \int_{\mathbb{R}} E\left[D_{t, x} F \mid \mathcal{F}_{t}\right] \tilde{N}(d t, d x)
$$

Remark 4.16 If $F \in L^{2}(\mu)$ is $\mathcal{F}_{T}$-measurable then $D_{t, x} F$ will in general belong to $\mathcal{G}^{*}$, but the smoothing property of the the conditional expectation with respect to $\mathcal{F}_{t}$ brings it down to $L^{2}(\lambda \times \nu \times \mu)$.

Next, define the generalized expectation of $F=\sum_{\alpha} c_{\alpha} K_{\alpha} \in(\mathcal{S})^{*}$ by

$$
E[F]=c_{0}
$$

and we say $F \in \mathcal{G}^{*}$ is $\mathcal{F}_{T}$-measurable if

$$
E\left[F \mid \mathcal{F}_{T}\right]=F
$$

We prove a $\mathrm{CHO}$ formula for $\mathcal{G}^{*}$. It is a Poisson random measure version of Theorem 4.14 in $[\mathrm{D} \emptyset \mathrm{P}]$.

\section{Theorem 4.17 (CHO theorem for $\mathcal{G}^{*}$ )}

Let $F \in \mathcal{G}^{*}$ be $\mathcal{F}_{T}$-measurable. Then

$$
F=E[F]+\int_{0}^{T} \int_{\mathbb{R}} E\left[D_{t, x} F \mid \mathcal{F}_{t}\right] \diamond \stackrel{\tilde{N}}{ }(t, x) \nu(d x) d t,
$$


where $E[F]$ denotes the generalized expectation of $F$.

Proof Let $F_{n} \in L^{2}(\mu)$ be $\mathcal{F}_{T}-$ measurable such that $F_{n} \rightarrow F$ in $\mathcal{G}^{*}$. Then there exists a $p$ such that $\left\|F_{n}-F\right\|_{\mathcal{G}_{-p+1}}^{2} \longrightarrow 0$ as $n \longrightarrow 0$. By Theorem 4.15 we obtain

$$
F_{n}=E\left[F_{n}\right]+\int_{0}^{T} \int_{\mathbb{R}} E\left[D_{t, x} F_{n} \mid \mathcal{F}_{t}\right] \diamond \dot{\widetilde{N}}(t, x) \nu(d x) d t \text { for all } n .
$$

For going to the limit in the last relation let us proceed as follows:

First we get that

$$
\left\|E\left[D_{t, x} \tilde{F} \mid \mathcal{F}_{t}\right] \diamond \dot{\widetilde{N}}(t, x)\right\|_{0,-(3 p+5)}^{2} \leq \text { const. } \cdot\left\|E\left[D_{t, x} \tilde{F} \mid \mathcal{F}_{t}\right]\right\|_{0,-3 p}^{2}\|\dot{\widetilde{N}}(t, x)\|_{0,-3}^{2}
$$

for $\tilde{F} \in \mathcal{G}^{*}$ with $\|\tilde{F}\|_{\mathcal{G}_{-p+1}}<\infty$. So it follows that

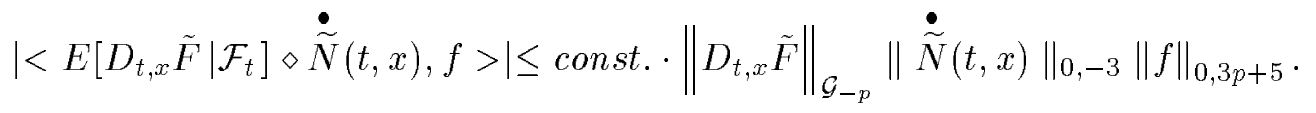

for $f \in(\mathcal{S})$. Thus this gives

$$
\begin{aligned}
& \left|<\int_{0}^{T} \int_{\mathbb{R}} E\left[D_{t, x} \tilde{F} \mid \mathcal{F}_{t}\right] \diamond \dot{\widetilde{N}}(t, x) \nu(d x) d t, f>\right| \\
& \leq \int_{0}^{T} \int_{\mathbb{R}}\left|<E\left[D_{t, x} \tilde{F} \mid \mathcal{F}_{t}\right] \diamond \dot{\widetilde{N}}(t, x), f>\right| \nu(d x) d t \\
& \leq \text { const. } \cdot\|f\|_{0,3 p+5}\left(\int_{0}^{T} \int_{\mathbb{R}}\left\|D_{t, x} \tilde{F}\right\|_{\mathcal{G}_{-p}}^{2} \nu(d x) d t\right)^{1 / 2}\left(\int_{0}^{T} \int_{\mathbb{R}}\|\dot{\widetilde{N}}(t, x)\|_{0,-3}^{2} \nu(d x) d t\right)^{1 / 2}
\end{aligned}
$$

Together with (4.11) we get the following inequality

$$
\left|<\int_{0}^{T} \int_{\mathbb{R}} E\left[D_{t, x} \tilde{F} \mid \mathcal{F}_{t}\right] \diamond \dot{\widetilde{N}}(t, x) \nu(d x) d t, f>\right| \leq \text { const. } \cdot\|f\|_{0,3 p+5}\|\tilde{F}\|_{\mathcal{G}_{-p+1}} .
$$

The last estimate implies that the expression

$$
\int_{0}^{T} \int_{\mathbb{R}} E\left[D_{t, x}\left(F_{n}-F\right) \mid \mathcal{F}_{t}\right] \diamond \stackrel{\dot{\widetilde{N}}}{ }(t, x) \nu(d x) d t
$$

converges to 0 in $(\mathcal{S})^{*}$ as $n \longrightarrow \infty$. The proof follows.

Finally we want to give an application of Theorem 4.15 to portfolios in financial markets. For this purpose we need the following extension of relation (4.5) to square integrable random variables. In this pure jump setting it is an extension from $D_{1,2}$ to $L^{2}(\mu)$ of Theorem 4.1 in [BDLØP]. 
Theorem 4.18 The minimal variance portfolio $\pi_{t}$ in the sense of Remark 4.4 is given by

$$
\pi_{t}=\frac{1}{\|x\|_{L^{2}(\nu)}^{2}} \int_{\mathbb{R}} x E\left[D_{t, x} F \mid \mathcal{F}_{t}\right] \nu(d x)
$$

for $F \in L^{2}(\mu)$, where $D_{t, x}$ is the stochastic derivative in Definition 4.9 .

Proof The proof follows from Theorem 4.15 and a Hilbert space argument, just as in the proof of Theorem 4.1 in [BDLØP]. We omit the details.

Example 4.18 We want to calculate the "closest" hedge to the digital option $F=$ $\chi_{[K, \infty)}(\eta(T)(\omega))$, given in Remark 4.5. Note, that $F \notin \mathbb{D}_{1,2}$. However, the minimal variance portfolio $\pi_{t}$ for $F$ can be still determined by means of relation (4.17). Now, take a sequence of continuously differentiable functions $0 \leq g_{n} \leq 1$ such that $g_{n} \longrightarrow \chi_{[K, \infty)}$ pointwise and such that $\frac{d}{d x} g_{n}$ has compact support for all $n$. It follows by Lemma 9 in [L] that $g_{n}(\eta(T)) \in \mathbb{D}_{1,2}$ and $D_{t, x} g_{n}(\eta(T))=g_{n}(\eta(T)+x)-g_{n}(\eta(T))$ for all $n$. Further, we have that $g_{n}(\eta(T)) \longrightarrow$ $\chi_{[K, \infty)}(\eta(T))=F$ and $g_{n}(\eta(T)+x) \longrightarrow \chi_{[K, \infty)}(\eta(T)+x)$ in $L^{2}(\mu)$. By Lemma 4.11 we know that there exists a subsequence such that $D_{t, x} g_{n_{k}}(\eta(T)) \longrightarrow D_{t, x} F$ in $\mathcal{G}^{*}$. On the other hand $D_{t, x} g_{n}(\eta(T)) \longrightarrow \chi_{[K, \infty)}(\eta(T)+x)-\chi_{[K, \infty)}(\eta(T))$ in $L^{2}(\mu)$. This implies that $D_{t, x} F=\chi_{[K, \infty)}(\eta(T)+x)-\chi_{[K, \infty)}(\eta(T))$. Together with (4.17) it follows that

$$
\begin{aligned}
\pi_{t} & =\frac{1}{\|x\|_{L^{2}(\nu)}^{2}} \int_{\mathbb{R}} x E\left[\chi_{[K, \infty)}(\eta(T)+x)-\chi_{[K, \infty)}(\eta(T)) \mid \mathcal{F}_{t}\right] \nu(d x) \\
& =\frac{1}{\|x\|_{L^{2}(\nu)}^{2}} E\left[\int_{0}^{1} \chi_{[K-x, K)}(\eta(T)) \frac{1}{x} d x \mid \mathcal{F}_{t}\right]
\end{aligned}
$$

So the "closest" hedge to $F$ is realized by the strategy

$$
\pi_{t}=\frac{-1}{\|x\|_{L^{2}(\nu)}^{2}} E\left[\chi_{(-\infty, K)}(\eta(T)) \log ((K-\eta(T)) \wedge 1) \mid \mathcal{F}_{t}\right], 0 \leq t \leq T .
$$

Acknowledgements We thank A. Løkka for suggestions and helpful comments. We are also grateful to G. Di Nunno and F. E. Benth for helpful remarks.

\section{References}

[AH-K] Albeverio, S., Høegh-Krohn, R., The Wightman axioms and the mass gap for strong interactions of exponential type in two-dimensional space time. J. Funct. Anal. 16, 39-82 (1974).

[AHPS] Albeverio, S., Hida, T., Potthoff, J., Streit, L., The vacuum of the Høegh-Krohn model as a generalized white noise functional, Phys. Lett. B 217, 511-514, (1989).

[AKS] Albeverio, S.,Kondratiev, Y.G., Streit, L., How to generalize White Noise Analysis to NonGaussian Spaces. In: Dynamics of Complex and Irregular Systems. Eds.: Ph. Blanchard et al., World Scientific (1993). 
[AØPU] Aase, K., Øksendal, B., Privault, N., Ubøe, J.: White noise generalizations of the ClarkHaussmann-Ocone theorem with application to mathematical finance. Finance and Stochastics $4,(2000), 465-496$.

[BG] Benth, F.E., Gjerde, J.: A remark on the equivalence between Poisson and Gaussian stochastic partial differential equations. Pot. Anal. 8, (1998), 179-193.

[BL] Benth, F.E., Løkka, A.: Anticipative calculus for Lévy processes and stochastic differential equations. Preprint series in Pure Mathematics, University of Oslo, 6, 2002.

[BDLøP] Benth, F.E., Di Nunno, G., Løkka, A., Øksendal, B., Proske, F.: Explicit representation of the minimal variance portfolio in markets driven by Lévy processes. Preprint series in Pure Mathematics, University of Oslo, 27, 2001.

[B] Bertoin, J.: Lévy Processes. Cambridge University Press, Cambridge 1996.

[BH] Bouleau, N., Hirsch, F.: Dirichlet Forms and Analysis on Wiener space. Walter de Gruyter, Berlin-New York, 1991.

[DØP] Di Nunno, G., Øksendal, B., Proske, F., White Noise Analysis for Lévy processes, Preprint series in Pure Mathematics, University of Oslo, 7, 2002.

[EvdH] Elliott, R., van der Hoek, J.: A general fractional white noise theory and applications to finance. Preprint, 2000.

[FSo] Föllmer, H., Sondermann, D.: Hedging of non-redundant contingent claims. Contributions to mathematical economics, 205-223, North Holland, Amsterdam 1986.

[GV] Gelfand, I. M., Vilenkin, N. Y.: Generalized Functions, Vol. 4: Applications of Harmonic Analysis. Academic Press (English translation), New York-London 1964.

[H] Hida, T.: White noise analysis and its applications. In: Proc. Int. Mathematical Conf., ed. by Chen, L.H.Y. Chen et al. North-Holland, Amsterdam, 43-48, 1982.

[HKPS] Hida, T., Kuo, H.-H, Potthoff, J., Streit, L.: White Noise. Kluwer, Dordrecht, 1993.

[HØ1] Hu, Y., Øksendal, B.: Fractional white noise calculus and applications to finance. Preprint 10/1999, University of Oslo.

[Hø2] Holden, H., Øksendal, B.: A white noise approach to stochastic differential equations driven by Wiener and Poisson processes. In M. Grosser et al. (editors): Nonlinear Theory of Generalized Functions, Chapman \& Hall/CRC 1999, pp 293-313.

[HøUZ] Holden, H., Øksendal, B., Ubøe, J., Zhang T.-S.: Stochastic Partial Differential EquationsA Modeling, White Noise Functional Approach. Birkhäuser, Boston 1996.

[I] Itô, K., Spectral type of the shift transformation of differential processes and stationary increments. Trans. Am. Math. Soc. 81, 253-263, 1956.

[JS] Jacod, J., Shiryaev, A.N., Limit Theorems For Stochastic Processes, Springer-Verlag, New York 1987. 
[Ka] Kachanowsky, N.A., On an analog of stochastic integral and Wick calculus in non-Gaussian infinite dimensional analysis, Methods Funct. Anal. Topology, 3 (3), 1-12, 1997.

[KDS] Kondratiev, Y., Da Silva, J. L., Streit, L.: Generalized Appell systems, Methods Funct. Anal. Topology, 3 (3), (1997), 28-61.

[KDSU] Kondratiev, Y., Da Silva, J. L., Streit, L., Us, G.: Analysis on Poisson and gamma spaces, Inf. Dim. Anal. Quant. Prob. Rel. Topics, 1 (1), (1998), 91-117.

[KO] Karatzas, I., Ocone, D.: A generalized Clark representation formula, with application to optimal portfolios. Stochastics and Stochastic Reports 34, (1991), 187-220.

[Ku $]$ Kuo, H. H., White Noise Distribution Theory. Prob. and Soch. Series, Boca Raton, FL: CRC Press, (1996).

[LSUV] Léon, J.A., Solé, J.L., Utzet, F., Vives, J.: On Lévy processes, Malliavin calculus and market models with jumps. Prepublications 2001, U.A.B., Barcelona. To appear in Finance and Stochastics.

[L] Løkka, A.: Martingale representation and functionals of Lévy processes. Preprint series in Pure Mathematics, University of Oslo, 21, 2001.

[M] Malliavin, P.: Stochastic Analysis. Springer-Verlag, New York 1997.

[N] Nualart, D.: The Malliavin Calculus and Related Topics. Springer, Berlin Heidelberg New York 1995.

[NS] Nualart, D., Schoutens, W.: Chaotic and predictable representations for Lévy processes. Stochastic Process. Appl., 90, (2000), 109-122.

[NV] Nualart, D., Vives, J., Anticipative calculus for the Poisson process based on Fock space. Séminaire de Probabilités XXIV, 154-165, (Lect. Notes Math., Vol. 1426.), Berlin: Springer $1988 / 89$.

[NZ] Nualart, D., Zakai, M.: Generalized stochastic integrals and the Malliavin calculus. Prob. Th. Rel. Fields 73, 255-280, 1986.

[O] Obata, N., White Noise Calculus and Fock Space, LNM, 1577, Berlin: Springer-Verlag, (1994).

[Ø] Øksendal, B.: An introduction to Malliavin calculus with applications to economics. Working paper, No 3/96, Norwegian School of Economics and Business Administration, 1996.

[Pi] Picard, J.: On the existence of smooth densities for jump processes. Prob. Th. Rel. Fields 105, (1996), pp. 481-511.

[PT] Potthoff, J., Timpel, M.: On a dual pair of smooth and generalized random variables. Potential Analysis 4, (1995), 637-654.

[P] Protter, P.: Stochastic Integration and Differential Equations. Springer-Verlag, Berlin 1990. 
[S] Schweizer, M.: A guided tour through quadratic hedging approaches. In: Jouini, E. et al. (editors), Option Pricing, Interest Rates and Risk Management, 538-574, Cambridge University Press, Cambridge 1999.

[Sa] Sato, K.: Lévy Processes and Infinitely Divisible Distributions, Cambridge University Studies in Advanced Mathematics, Vol. 68, Cambridge University Press, Cambridge 1999.

[T] Thangavelu, S.: Lectures on Hermite and Laguerre Expansions. Mathematical Notes 42, Princeton University Press 1993.

[Ü1] Üstünel, A.S.: Representation of distributions on Wiener space and stochastic calculus of variations. J. Functional Analysis, 70, (1987), 126-139.

[Ü2] Üstünel, A.S.: An Introduction to Analysis on Wiener Space. (Lecture Notes in Mathematics, No 1610). Springer, Berlin Heidelberg New York 1995. 